\title{
ON THE BEHAVIOR OF AN ANALYTIC FUNCTION ABOUT AN ISOLATED BOUNDARY POINT
}

\author{
MAKOTO OHTSUKA*
}

Introduction. Let $D$ be an open set in the $z$-plane, $C$ its boundary, $z_{0}$ a point on $C$, and $f(z)$ a one-valued meromorphic function in $D$. Given a set $E \subset D+C$, we denote the intersection of $E$ with $G_{r}=\left\{0<\left|z-z_{0}\right|<r\right\}$ by $E_{r}$, and the set of values $\left\{f(z) ; z \in D_{r}\right\}$ by $f\left(D_{r}\right)$. The cluster set $S_{z_{0}}^{(D)}$ of $f(z)$ at $z_{0}$ in $D$ is defined by $\bigcap_{r}\left[f\left(D_{r}\right)\right]^{a}$, where []$^{a}$ denotes the closure of the set in [ ], and the range of values $R_{z_{0}}^{(D)}$ is defined by $\bigcap f\left(D_{r}\right)$. Further the cluster set $S_{z_{v}}^{(F)}$ on $E$ is defined by $\cap\left[\bigcup_{r} S_{z}^{(D)}\right]^{a}$, where $S_{z}^{(D)}$ at an inner point $z$ is put equal to $f(z)$. In the theory of cluster sets relations between $S_{z_{0}}^{(D)}, S_{z_{0}}^{(C)}, R_{z_{0}}^{(D)}$ are pursued chiefly. ${ }^{1)}$ Here we refer to the following two principal theorems under the assumption that $z_{0}$ is non-isolated:

(I) $\left(\right.$ Brelot $\left.^{2)}\right) .\left(S_{z_{0}}^{(D)}\right)^{b} \subset S_{z_{0}}^{(C)}$, where ()$^{b}$ denotes the boundary of the set in () .

(II) (Kunugui [5]). Each component of $S_{z_{0}}^{(D)}-S_{z_{0}}^{(C)}$, with two possible exceptions, is contained in $R_{z_{0}}^{(D)}$, provided that $D$ is a domain. ${ }^{3)}$

It is always assumed that $z_{0}$ is non-isolated in these theorems, and the case when $z_{0}$ is isolated is left to the well-known Picard's theorem.

Above the cluster sets are defined for a function which takes values in a plane. However, the definitions can be generalized to a function, which is defined in a plane domain and takes values on an abstract Riemann surface, and

Received November 7, 1951.

* This work was done by the writer as a fellow of the Yukawa Foundation of Osaka University.

1) For various results and literatures, cf. [7].

2) See [2], Theorem in $\$ 6$. The form of Brelot's theorem is different from (I), but the equivalency is proved as usual. Cf. [6], for instance.

3) This theorem can be proved also in the case where $D$ is any open set as follows: Suppose that there exists a component $\Omega$ of $S_{z_{0}}^{(D)}-S_{z_{0}}^{(C)}$, at least three points of which do not belong to $R_{z_{0}}^{(D)}$. Let $w_{0}$ be such an exceptional value. Since $w_{0} \in S_{z_{0}}^{(D)}$, we can choose $\left\{z_{n}\right\}, z_{n} \rightarrow z_{0}$, such that $f\left(z_{n}\right) \rightarrow w_{0}$. Among the inverse images in $D$ of the segments $\left\{\overline{\left.f\left(z_{n}\right) w_{0}\right\}}\right.$ in $\Omega$, we can find an inverse image $l$ in $D$ terminating at $z_{0} . f(z)$ has a limit $w_{1} \in \Omega$ as $z \rightarrow z_{0}$ along $l$. Let $D_{1}$ be the component of $D$ which contains $l$, and $C_{1}$ its boundary. Then $S_{z_{0}}^{\left(D_{1}\right)}$ contains $w_{1}$, and $S_{z_{0}}^{(n)} \supset S_{z_{0}}^{\left(D_{1}\right)}, S_{z_{0}}^{(C)} \supset S_{z_{0}}^{\left(C_{1}\right)}, R_{z_{0}}^{(D)} \supset R_{z_{0}}^{\left(D_{1}\right)}$. The component $\Omega_{1}$, which contains $w_{1}$, of $S_{z_{0}}^{\left(D_{1}\right)}-S_{z_{0}}^{\left(C_{1}\right)}$ includes $\Omega$ by (I). Hence $R_{z_{0}}^{\left(\rho_{1}\right)}$ does not contain at least three values in $\Omega_{1}$. This is contrary to (II). 
some results are obtained (cf. [8], Chap. V, §1). In this note we shall investigate the behavior of such an analytic function about an isolated boundary point by making use of the methods in the theory of cluster sets.

1. Let $D$ be a domain in the $z$-plane, $z_{0}$ its isolated boundary point, $\mathfrak{R}$ an abstract Riemann surface in the sense of Weyl-Radó, and $f(z)$ an analytic function mapping $D$ into $\Re$. Setting $\left\{0<\left|z-z_{0}\right|<r\right\}=G_{r}$ and $D \cap G_{r}=D_{r}$, we denote the set of values $\left\{f(z) ; z \in D_{r}\right\}$ by $\mathscr{D}_{r}$. The cluster set $S_{z_{0}}^{(D)}$ of $f(z)$ in $D$ at $z_{0}$ is defined by $\bigcap_{r} \mathscr{D}_{r}^{a}$, where $\mathscr{D}_{r}^{a}$ is the closure taken relatively to $\Re$ of $D_{r}$, and the range of values $R_{z_{0}}^{(D)}$ is defined by $\bigcap_{r} \mathfrak{D}_{r .}{ }^{4)}$

We begin with the following lemma:

LEMma. Suppose that the cluster set $S_{z_{0}}^{(D)}$ is not empty. Then $S_{z_{0}}^{(D)}$ consists of either a point on $\mathfrak{R}$ or $\mathfrak{\Re}$ itself.

Proof. Suppose that the assertion is not true. Then there is a neighborhood $N$ on $\Re$ of a boundary point $P_{0}$ of $S_{z_{0}}^{(n)}$ such that $S_{z_{0}}^{(n)} \nsubseteq N^{a}$. Let $\Delta:|t|<1$ be a local parameter circle, corresponding to $N$ and with $t=0$ as the image of $P_{0}$. Consider the inverse image $D_{1}$ in $D$ of $N$, and denote the composed function $t(f(z))$ in $D_{1}$ by $t(z)$. Since $P_{0} \in S_{z_{0}}^{(D)}$, we can find a sequence $\left\{z_{n}\right\}$ tending to $z_{0}$ such that $f\left(z_{n}\right) \rightarrow P_{0}$. Hence $z_{0}$ is a boundary point of $D_{1}$. Further $z_{0}$ is not isolated, because there is a sequence $\left\{z_{n}^{\prime}\right\}, z_{n}^{\prime} \rightarrow z_{0}$, outside $D_{1}$ such that $f\left(z_{n}^{\prime}\right)$ tends to a certain point of $S_{z_{0}}^{\text {inj }}$ outside $N$. Thus $D_{1}$ is an open subset of $D$, with $z_{0}$ as its non-isolated boundary point. The cluster set of $t(z)$ on the boundary of $D_{1}$ at $z_{0}$ consists of points on $|t|=1$ but does not contain $t=0$, whereas this point belongs to the boundary of the cluster set of $t(z)$ in $D_{1}$ at $\dot{z}_{0}$. This contradicts $(I)$ in the introduction.

2. Let us suppose first that $\mathfrak{R}$ is of genus finite. $\mathfrak{R}$ is then conformally

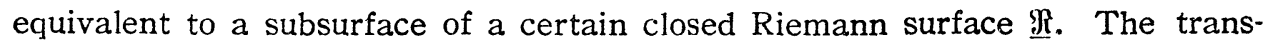
formed function. which takes values on $\Re$, of $f(z)$ will be denoted by $F(z)$. We shall use notations $\underline{S}_{z_{0}}^{(D)}$ and $\underline{R}_{z_{0}}^{(D)}$ to represent the cluster set and the range of values of $F(z)$ respectively. Since $\underline{S}_{z_{0}}^{(D)}$ is non-empty, it consists of a point on $\mathfrak{R}$ or of $\stackrel{\Re}{n}$ itself by the above lemma.

In case $\underline{S}_{z_{0}}^{(\prime)}$ consists of one point on $\Re$, the image $\mathscr{D}_{r}$ on $\Re$ of $D_{r}$ converges to an inner point of $\Re$ or to a parabolic ideal boundary component of $\Re$ as $r \rightarrow 0 .^{5}$

The case in which $\underline{S}_{z_{0}}^{(n)}=\mathfrak{R}$ will be investigated in details in the sequel. We shall denote the genus of $\underline{\Re}$ by $\underline{p}$.

Case: $\underline{p}=0$. We suppose that $\underline{R}-\underline{R}_{z_{0}}^{(D)}$ contains at least three points,

4) Notice that $f(z), S_{z_{0}}^{(D)}$ and $R_{z_{0}}^{(D)}$ take values on a Riemann surface here, though the same notations as in the introduction are used.

5) As for the definition of a parabolic ideal boundary component, see [8], Chap. III, $\S 5$. 
say, $\underline{P}_{1}, \underline{P}_{2}, \underline{P}_{3}$. Since $\underline{P}_{1} \in \underline{S}_{z_{0}}^{(D)}$, there is a sequence $\left\{z_{n}\right\}$ tending to $z_{0}$ such that $F\left(z_{n}\right) \rightarrow \underline{P}_{1}$. Connect every $F\left(z_{n}\right)$ with $\underline{P}_{1}$ by a curve $L_{n}$ such that $L_{n}$ approaches $\underline{P}_{1}$ as $n \rightarrow \infty$. For a sufficiently large number $n_{0}$ the inverse image $l_{n_{0}}$ with $z_{n_{0}}$ as its starting point must lie near $z_{0}$ and hence terminate at $z_{0}$, because $\left\{F(z) ; z \in l_{n}\right\} \subset L_{n} \rightarrow \underline{P}_{1}$ as $n \rightarrow \infty$. A part $D_{0}$ of $D$, near $z_{0}$ and cut by $l_{n_{0}}$, can be regarded as an angular domain with the opening $2 \pi . F(z)$ tends to a value $\underline{P}_{0} \in L_{n_{0}}$ as $z \rightarrow z_{0}$ on $l_{n_{0}}$. Since $F(z) \neq \underline{P}_{1}, \underline{P}_{2}, \underline{P}_{3}$, near $z_{0}, F(z)$ tends to $\underline{P}_{0}$ uniformly as $z \rightarrow z_{0}$ in $D_{0}$ by Lindelöf-Iversen's theorem [3]. Thus $\underline{S}_{z_{0}}^{(D)}=\left\{\underline{P}_{0}\right\}$, and a contradiction is lead. Therefore when $\mathscr{R}$ is of genus zero and $\underline{S}_{z_{0}}^{(D)}=\mathfrak{R}$, then $\underline{R}_{z_{0}}^{(D)}$ contains all points of $\underline{\Re}$ with two possible exceptions. This fact is none other than Picard's theorem.

Case: $\underline{p}=1$. Suppose that $\underline{R}_{z_{0}}^{(D)} \neq \underline{S}_{z_{0}}^{(D)}=\underline{\Re}$, and take a point $\underline{P} \in \underline{\Re}-\underline{R}_{z_{0}}^{(D)}$. In the mapping of the universal covering surface $\Re^{\infty}$ of $\Re$ onto the finite whole $w$-plane, $\underline{P}_{1}$ corresponds to an enumerably infinite number of points in the plane. Similarly as in the preceding case we get a curve $l$ terminating at $z_{0}$ such that $F(z)$ tends to a value $\underline{P}_{0}$ on $\underline{R}$ as $z \rightarrow z_{0}$ along $l$. In the angular domain $D_{0}$ cut by $l$, any branch $w(z)$ of the composed function $w(F(z))$ becomes one-valued regular by monodromy theorem. It tends to respective definite limits along both sides of $l$ and does not take near $z_{0}$ the $w$-values corresponding to $\underline{P}_{1}$. Hence $w(z)$ tends to a certain value uniformly in $D_{0}$ by Lindelöf-Iversen's theorem. This shows $\underline{S}_{z_{0}}^{(D)}=\left\{\underline{P}_{0}\right\}$, contrary to the assumption that $\underline{S}_{z_{0}}^{(D)}=\underline{\Re}$. Thus, when $\underline{\Re}$ is of genus one and $\underline{S}_{z_{0}}^{(D)}=\underline{\Re}$, then $\underline{R}_{z_{0}}^{(D)}=\underline{\Re}$.

Case: $\underline{p} \geq 2$. On mapping $\mathfrak{\Re}^{\infty}$ onto $|w|<1$ it is shown from $\underline{S}_{z_{0}}^{(D)}=\Re$ as above that $\underline{R}_{z_{0}}^{(D)}=\underline{\Re}$. $\underline{\Re}$ is made of planar character by $\underline{p}$ disjoint simple closed curves $\left\{C_{i}\right\}(i=1,2, \ldots, \underline{p})$. By connecting infinitely many samples along the opposite shores of $\left\{C_{i}\right\}$, we obtain a Schottky covering surface $\bar{\Re}$, of planar character and having no relative boundary, over $\mathfrak{\Re}$. $\overline{\mathfrak{R}}$ is mapped conformally onto a domain outside a perfect set $F$ in the $w$-plane and any image of $C_{i}$ is a closed curve. For any $\underline{P}_{1} \in C_{1}$ there exists a sequence $\left\{z_{n}\right\}$ tending to $z_{0}$ such that $F\left(z_{n}\right)=\underline{P}_{1}$. We may suppose that on $C_{1}$ there is no image of a double point of $F(z)$. We denote by $C_{1}^{\prime}$ a conjugate curve, which intersects $C_{1}$ merely at $\underline{P}_{1}$ and on which no image of a double point lies. Let $l_{n}$ be the inverse inage through $z_{n}$ of $C_{1}$. If no $l_{n}$ terminates at $z_{0}$, there exists a number $n_{0}$ such that every $l_{n}$ for $n \geqslant n_{0}$ is a simple closed curve around $z_{0}$, because disjoint inverse images of $C_{1}$ can not cluster in $D$ and no image is a closed curve surrounding a compact domain in $D$. Consider the inverse image $l_{n_{0}}^{\prime}$ of $C_{1}^{\prime}$, which starts from $z_{n_{0}}$ and runs inside $l_{n_{0}}$. A domain near and inside $l_{n_{0}}$ corresponds to one side of $C_{1}$ on $\mathfrak{R}$. Therefore $l_{n_{0}}^{\prime}$ can not intersect $l_{n_{0}}$ again and hence must terminate at $z_{0}$. Thus the inverse image through $z_{n}$ of $C_{1}$ or $C_{1}^{\prime}$ terminates at 
$z_{0}$ for any large $n$. Without loss of generality we may suppose that an image $l$ of $C_{1}$ terminates at $z_{0}$. In the angular domain $D_{0}$ cut by $l$, any branch $w(z)$ of the composed function $w(F(z))$ becomes one-valued regular. Its cluster sets $S_{1}$ and $S_{2}$ on the both sides of $l$ at $z_{0}$ lie either on one and the same image $I$ of $C_{1}$ or on two images $\Gamma_{1}$ and $\Gamma_{2}$ of $C_{1}$ respectively. In the former case $S_{1} \cap S_{2}$ is not empty and the cluster set $S$ of $w(z)$ at $z_{0}$ in $D_{0}$ coincides with $S_{1} \cup S_{2}$ on account of (I), (II), because $w(z)$ does not take values of the perfect set $F$ whose points lie both outside and inside $\Gamma$. Hence $\underline{S}_{z_{0}}^{(D)} \subset C_{1}$, but this contradicts the assumption: $\underline{S}_{z_{0}}^{(D)}=\mathfrak{R}$. The latter case is impossible too by (I), (II), because $S$ is a continuum but every component of the complement of $\Gamma_{1} \cup \Gamma_{2}$ contains points of $F$. Hence it does not arise that $\underline{S}_{z_{0}}^{(p)}=\underline{\Re}$ for $\underline{\mathfrak{R}}$ of genus $\underline{p} \geqslant 2$.

We have considered so far the case when the genus of the original $\mathfrak{R}$ is finite. Finally we suppose that $\Re$ is of genus infinite. If there is $r>0$ such that $\mathscr{D}_{r}$ is of genus finite, the foregoing discussions apply. Consequently we suppose that every $\mathfrak{D}_{r}$ is of genus infinite. We can then take a mutually nonhomotopic disjoint infinite sequence of loop cuts $\left\{C_{n}\right\}, C_{n} \subset \mathfrak{D}_{1 / n}$, such that $C_{n}$ does not divide $\Re$ and approaches the ideal boundary of $\Re$ as $n \rightarrow \infty$. As in the preceding case we find an inverse image, which terminates at $z_{0}$, of a certain $C_{n}$ or its conjugate loop cut $C_{n}^{\prime}$. The cluster set of $f(z)$ along it is contained in $C_{n}$ or $C_{n}^{\prime}$ and hence non-empty. Accordingly by Lemma in $\S 1 S_{z_{0}}^{(D)}=\Re$. By considering the Schottky covering surface of $\mathfrak{R}$ a contradiction will be lead as before.

We now summarize the results in the following:

Theorem 1. Let $f(z)$ be a function, which is defined in a plane domain $D$ with an isolated boundary point $z_{0}$ and takes values on an abstract Riemann surface $\mathfrak{*}$. Then either the image of the ring domain $G_{r}: 0<\left|z-z_{0}\right|<r$ contained in $D$ converges to an inner point of $\Re$ or to a parabolic ideal boundary component of $\mathfrak{R}$ as $r \rightarrow 0$, or the range of values of $f(z)$ in $D$ at $z_{1}$ is conformally equivalent to a sphere with two possible exceptions or to a torus.

It is easy to find functions which realize these cases.

3. When $\mathfrak{R}$ is of genus finite, Theorem 1 can be proved also by Ahlfors' theory of covering surfaces [1]. We shall give an outline of the proof.

Since there exists a one-valued non-constant meromorphic function on $\underline{R}$ of $\S 2, \mathfrak{R}$ is conformally equivalent to a subsurface of a closed surface $\mathfrak{R}_{\sigma}$, which covers the Riemann sphere $\sigma$ touching the $w$-plane at $w=0$ and with diameter of length 1. Denoting the composed function $w(f(z))$ by $w(z)$, we consider the Riemann surface $\bar{\Re}_{w}$ of the inverse function of $w(z)$. If $z=0$ is removable for $w(z)$, the image on $\Re_{\sigma}$ of $G_{r}$ converges to a point on $\Re_{\sigma}$. The image on $\mathfrak{R}$ of $G_{r}$ converges then to a point or to a parabolic ideal boundary component of $\mathfrak{R}$. 
Hence suppose that $z=0$ is an essential singularity of $w(z)$. Similarly as for Riemann surfaces of parabolic type, it is seen that $\bar{\Re}_{w}$ is regularly exhaustible. Regard now $\overline{\mathfrak{R}}_{w}$ as a covering surface over $\mathfrak{R}_{\sigma}$ and denote it by $\overline{\mathfrak{R}}_{\sigma}$. Then $\bar{\Re}_{\sigma}$ is still a regularly exhaustible covering surface over $\mathfrak{R}_{\sigma}$, because the closed surface $\mathfrak{R}_{\sigma}$ covers $\sigma$ only in finite times.

On the other hand, if the genus of $\Re_{0}$ is $q \gtrsim 2$, Ahlfors' fundamental inequality gives

$$
0=\stackrel{+}{\rho} \text { 르 }(2 q-2) S(r)-h L(r),
$$

where the usual notations are used; especially, $S(r)$ is the average covering number over $\mathfrak{R}_{\sigma}$ of the part of $\bar{\Re}_{\sigma}$ corresponding to $D-G_{r}^{a}$. Hence

$$
\frac{L(r)}{S(r)} \geq \frac{2 q-2}{h}>0,
$$

which contradicts the fact that $\bar{\Re}_{\sigma}$ is regularly exhaustible.

Next suppose that $\mathfrak{R}_{\sigma}$ is of genus one. If there is a number $r_{0}>0$ such that the part $\bar{\Re}_{\sigma}^{\prime}$ of $\bar{\Re}_{\sigma}$ corresponding to $G_{r_{0}}$ does not cover a point $P_{0}$ of $\Re_{\sigma}$, regard $\bar{\Re}_{\sigma}^{\prime}$ as a covering surface over $\mathfrak{R}_{\sigma}^{\prime}=\mathfrak{R}_{\sigma}-\left\{P_{0}\right\}$. Applying Ahlfors' inequality to them, there follows $L(r) / S(r) \geqslant 1 / h>0$, which contradicts the regular exhaustibility of $\bar{\Re}_{o}^{\prime}$. As is known, Picard's theorem is proved by the same method.

It is not comprehensible to me, however, how such a method can be utilized in the case when $\mathfrak{R}$ is of genus infinite.

4. In [8], Chap. III, $\S 6$, the following theorem was proved:

ThEOREм 2. Let $\mathfrak{R}$ be an abstract Riemann surface with universal covering surface $\mathfrak{H}^{\infty}$ of hyperbolic type. In the mapping of $\mathfrak{H}^{\infty}$ onto $U:|z|<1$, the parabolic ideal boundary components of $\mathfrak{R}$ and the classes of parabolic fixed points, equivalent with respect to a Fuchsian group, on $\Gamma:|z|=1$ correspond to each other in a one-to-one manner.

The proof in [8] was different from the usual one given for a plane domain (e.g., [4], pp. 31-34). But once Theorem 1 is established, Theorem 2 can be proved in the usual way.

\section{BIBLIOGRAPHY}

[1] L. Ahlfors: Zur Theorie der Überlagerungsfächen, Acta Math., 65 (1935), pp. 157-194.

[2] M. Brelot: Sur l'allure à la frontière des fonctions harmoniques, sousharmoniques ou holomorphes, Bull. Soc. Roy. Liège, (1939), pp. 468-477.

[3] F. Iversen: Recherches sur les fonctions inverses des fonctions méromorphes, Thèse de Helsingfors, (1914).

[4] G. Julia: Leçons sur la représentation conforme des aires multiplement connexes, Paris (1934). 
[5] K. Kunugui: Sur un problème de M. A. Beurling, Proc. Imp. Acad. Tokyo, 16 (1940), pp. 345-350.

[6] K. Noshiro: On the singularities of analytic functions, Jap. Journ. Math., 17 (1940), pp. $37-96$.

[7] K. Noshiro: A theorem on the cluster sets of pseudo-analytic functions, Nagoya Math. Journ., 1 (1950), pp. 83-89.

[8] M. Ohtsuka: Dirichlet problems on Riemann surfaces and conformal mappings, Nagoya Math. Journ., 3 (1951), pp. 91-137.

Mathematical Institute,

Nagoya University 